\title{
A Survey of Internal Medicine Residents: Their Learning Environments, Bias and Discrimination Experiences, and Their Support Structures
}

\author{
Sylk Sotto-Santiago $\mathbb{D}^{\prime}$ \\ Jacqueline $\mathrm{Mac}^{2}$ \\ James Slaven ${ }^{3}$ \\ Maria Maldonado ${ }^{4}$ \\ 'Department of Medicine, Indiana \\ University School of Medicine, \\ Indianapolis, IN, USA; ${ }^{2}$ Department of \\ Counseling and Higher Education, \\ Northern Illinois University, Dekalb, IL, \\ USA; ${ }^{3}$ Department of Biostatistics, \\ Indiana University School of Medicine, \\ Indianapolis, IN, USA; ${ }^{4}$ Department of \\ Medicine, Icahn School of Medicine at \\ Mount Sinai, New York, NY, USA
}

Purpose: While there is an emerging body of literature that demonstrates how racism and bias negatively impact the experiences of physicians and trainees from underrepresented groups in medicine in the US, little is known about the experiences of internal medicine trainees and their learning environments. The purpose of this study was to examine these learning environments and explore trainees' perceptions of race/ethnicity-related topics.

Methods: A 35-item confidential electronic survey was disseminated to trainees from 11 internal medicine training programs in the US. A total of 142 trainees participated. Purposive sampling ensured alignment with 2018 IM trainee demographics by sex, race and ethnicity. Analyses were performed including chi-square, Fisher's exact tests, and logistic regression. Results: Key findings reveal $63 \%$ of respondents perceived disparities in the care provided to diverse patients. Two in three respondents were confident that their institution would respond to discrimination, but only $1 / 3$ of respondents perceived appropriate reporting mechanisms. Black/African American trainees reported needing to minimize aspects of their race and were less likely to perceive their institutions as being supportive to people of color.

Conclusion: Access to timely information about trainees' experiences with discrimination and bias in graduate medical education is imperative to disrupt systemic racism and health inequities. Findings suggest a perceived difference in health care provided to minoritized groups, a gap in formal mechanisms for reporting racism and discrimination experienced by trainees, and environments that challenge a sense of belonging. Findings add to current literature exposing the experience of underrepresented trainees in the US.

Keywords: discrimination, bias, graduate medical education, disparities, underrepresented

\section{Introduction}

The importance of a diverse healthcare workforce towards enhancing culturally competent care, mitigating healthcare disparities, and ensuring a cadre of physicians dedicated to caring for a diverse patient population is hard to dispute. ${ }^{1-7}$ Residents and fellows, especially from underrepresented groups (URGs), comprise an essential population to develop and strengthen as a pipeline of culturally competent physicians.

There is evidence that URGs in medicine experience prejudice, bias, and discrimination based on their gender, gender expression or identity, sexual orienta-

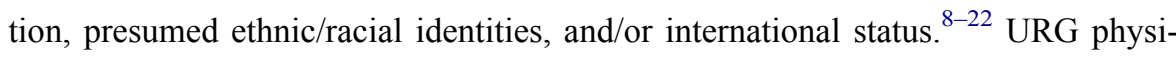
cians also report patients rejecting their care, patients and colleagues mistaking them for non-physician employees, and being held to higher performance standards.
Correspondence: Sylk Sotto-Santiago Department of Medicine, Indiana University School of Medicine, 545 Barnhill Dr. Emerson Hall 305, Indianapolis, IN, 46202, USA

Email ssotto@iu.edu 
For example, scholars have reported psychological abuse and discrimination based on gender and sexual harassment are commonly experienced by residents in training programs. ${ }^{9}$ More specifically, Black physicians face complex social and emotional challenges during training including discrimination, differing expectations, social isolation, and consequences. Participants in several studies have reported Black colleagues reprimanded more harshly for the same transgression as their White colleagues and perceived to perform at lower levels than White counterparts. ${ }^{10}$ Other studies that have centered on international medical graduates' experience in the US have also highlighted self-reported considerable bias and prejudice. $^{12}$ Unfortunately, these instances have not greatly declined over time. ${ }^{13}$ A recent study described the experiences of underrepresented resident/trainees found that participants described three major themes: a daily barrage of microaggressions and bias, minoritized trainees tasked as race/ethnicity ambassadors, and challenges negotiating professional and personal identity while seen as "other". ${ }^{20}$ In higher education literature, "othering" is the process of treating or perceiving one as different from ourselves; treating individuals as lesser based on implicitly perceived differences.

The literature seems to share a call for direct approaches to address such issues by creating supportive networks and raising awareness. In addition, scholars have called for policies, procedures, and structural changes that promote cultural change within academic institutions. These experiences, combined with unsupportive structures, indicate a strong need for institutional mechanisms that effectively address and respond to racism, bias, and discrimination.

While this emerging literature portrays how racism, bias, and discrimination negatively impacts the success and experiences of physicians, learners, and trainees from URGs, little is known about the specific experiences of internal medicine (IM) residents and how their learning environment eliminates, perpetuates, or buffers these types of experiences. Gaps in the literature demonstrate a limited assessment of resident learning environments. ${ }^{8}$ Because Internal medicine represents the largest training specialty offering $25 \%$ of all intern positions in the National Resident Match Program and is the frontline of diagnoses and treatments of patients, it is important to understand what these trainees face. ${ }^{23}$ Moreover, about $57 \%$ of internal medicine primary care residency graduates go directly into primary care, an important specialty towards achieving health equity. ${ }^{24}$
Understanding the current learning environment for trainees is crucial to the creation of necessary support and patient care structures to facilitate an inclusive excellent environment for all. Hence, our examination of the learning environments of internal medicine residents with a special focus on the URG experience.

\section{Methods}

Two research questions guided this survey study: (1) Do internal medicine trainees perceive their learning environment, programmatic and institutional structures responsive to issues of racism, bias, and discrimination? (2) To what extent do residents from URGs perceive their learning environment to be welcoming and inclusive?

The survey was developed with scholars in equity, diversity, inclusion, and antiracism. It also included experts in graduate medical education and survey design who reviewed the survey for content validity. Survey questions were drawn from previously validated tools that addressed cultural engagement, such as the Culturally Engaging Campus Environments (CECE), Workplace Discrimination, and Racial and Ethnic Microaggressions Scale (REMS). ${ }^{14,17,25}$ CECE was developed by the National Institute for Transformation and Equity, to assess and understand institutions' campus environments and to maximize success among diverse higher education students. The CECE Survey includes 9 indicators under two constructs: cultural relevance and cultural responsiveness. ${ }^{25}$ Workplace discrimination assisted in exploring discrimination among practicing physicians. RMES measures racial microaggressions as subtle statements and behaviors that unconsciously communicate denigrating messages to people of color. ${ }^{14}$ The final instrument also included demographic information and internal medicine residency type. Per survey research techniques, cognitive interviews and preliminary testing were performed in order to examine the final instrument's item comprehension, judgment, and response. ${ }^{26}$ Indiana University Institutional Review Board (IU IRB) reviewed this study and considered it exempt (protocol number: 1802080330, exemption granted on $3 / 5 / 2018$ ). The IU IRB determined this study exempt given its minimum to no risk to human participants as an anonymous survey and per regulatory category: research studying educational practices.

The survey was distributed electronically to IM program directors at eleven IM residencies nationwide to distribute survey invitations to their residents during the summer of 2018. These residency programs represented several regions, types of programs, and ensured that 
Table I Demographics Table

\begin{tabular}{|c|c|c|c|c|c|}
\hline \multicolumn{6}{|c|}{ ACGME Active Residents Self-Identification by Sex and Ethnicity } \\
\hline Gender & ACGME* & Study & Ethnicity & ACGME* & Study \\
\hline Women & $39.9 \%$ & $48.2 \%$ & White & $48.4 \%$ & $47.2 \%$ \\
\hline Men & $54.2 \%$ & $51.9 \%$ & Asian/Pacific Islander & $35.7 \%$ & $29.6 \%$ \\
\hline \multirow[t]{3}{*}{ Not Reported } & \multirow[t]{3}{*}{$5.9 \%$} & & Hispanic/Latino/a/x & $8.6 \%$ & $10.2 \%$ \\
\hline & & & Black/African American & $7.0 \%$ & $5.6 \%$ \\
\hline & & & Native American/Alaskan Native & $0.1 \%$ & $\mathrm{n} / \mathrm{a}$ \\
\hline \multicolumn{3}{|c|}{$\begin{array}{l}\text { US Regions: Northeast, Midwest, South, } \\
\text { Mountain. }\end{array}$} & * ACGME Data Resource Book 2017-2018, \% based on self-identification data & & \\
\hline
\end{tabular}

resident sample mirror current Accreditation Council for Graduate Medical Education (ACGME) IM resident demographic data (Table 1). For this analysis, we focus on findings related to race/ethnicity and gender.

Data analysis began with descriptive statistics. Additional analyses were performed to determine if there were significant differences in responses between genders and between races/ethnicities. Chi-Square tests were used to determine if there was significant heterogeneity in responses, with Fisher's Exact tests being used when cell counts were low. Logistic regression analyses were then performed to generate odds ratios, using White participants as the reference category for race and men for gender. Although we did not have adequate power to perform a multivariable analysis with an interaction term, we did analyze race associations for women and men separately, to determine if there were different patterns. Analyses were performed to determine if there were significant differences between the analysis group and the excluded group, with no significant heterogeneity found. In this article, we focus on the most salient results related to inclusive learning and work environments, experiences with discrimination and bias, and institutional support structures. All analytic assumptions, including proportional odds for multi-level response questions, were verified and analyses were performed using SAS v9.4 (SAS Institute, Cary, NC).

\section{Results}

A total of 142 respondents completed the survey out of an estimated potential 1100 trainees. The average in-app and online survey response rate ranged from $13-20 \%$. As an external online survey, we aimed to reach at least $10 \%$ of the sample population, exceeding recommendations by Johnson \& Brooks (2010) and Gliner, Morgan, and
Leech (2009). ${ }^{27,28}$ A higher response rate was pursued by reminder notices and increasing the open period of the survey. Purposive sampling ensures that the sample represented the qualities and demographics possessed by current IM nationwide. The responses are representative of the sample based on ACGME demographic data from 2018 (Table 1). A study by Fosnacht et. al. $(2017)^{29}$ did not find that a $5 \%$ response rate or even a $75 \%$ response rate provides unbiased population estimates under all circumstances, but rather that additional effort to move response rates marginally higher will frequently only shift survey results in trivial ways. This seems especially the case when the sample represents a population. Table 2 summarizes these results.

\section{Inclusive Learning and Work Environments}

The most salient racial relationships indicated that Black/ African American (AA) were 9.5 times more likely than White respondents to report needing to "minimize aspects" of their race including language, clothes, style, and ways of interacting in order to be able to "fit in" to the culture of the program/institution; 25 times more likely than White respondents to feel isolated at the institution; 7 times more likely than White respondents to feel like they need to work twice as hard to get the same treatment. Those who identified as Hispanic/Latino/a/ $\mathrm{x}$ indicated they were nearly 5 times less likely to be unfairly humiliated in front of others.

Women indicated they were 179 times more likely to report being viewed not as a physician, were 2.2 times more likely to have been unfairly humiliated, 5.4 times as likely to feel the need to work twice as hard, and 4.6 times more likely to feel isolated. 
Table 2 Summary of Likelihood

\begin{tabular}{|l|l|l|}
\hline Metric & Demographic & Likelihood (+) More Likely, (-)Less Likely \\
\hline Minimize themselves & Black/AA vs White & $+9.5 \mathrm{X}$ \\
\hline \multirow{2}{*}{ Isolation } & Black/AA vs White & $+25 \mathrm{X}$ \\
\cline { 2 - 3 } & Women vs Men & $+4.6 \mathrm{X}$ \\
\hline \multirow{2}{*}{ Feel the need to work harder } & Black/AA vs White & $+7 \mathrm{X}$ \\
\cline { 2 - 3 } & Women vs Men & $+5.4 \mathrm{X}$ \\
\hline \multirow{2}{*}{ Unfairly humiliated in front of others } & Hispanic/Latino/a/X vs White & $-5 X$ \\
\cline { 2 - 3 } & Women vs Men & $+2.2 \mathrm{X}$ \\
\hline \multirow{2}{*}{ Not viewed as a physician } & Women vs Men & $+179 X$ \\
\hline Report experiences of discrimination by patients/families & Women vs Men & $+6.2 X$ \\
\cline { 2 - 3 } & Black/AA vs White & +3.2 \\
\hline
\end{tabular}

\section{Experiences with Discrimination and Bias}

Over $16 \%$ of participants reported experiencing discrimination from faculty and/or administration. Nearly three times as many respondents $(46.3 \%)$ indicated that they experienced discrimination from patients and families. Of those who indicated "yes", $70.0 \%$ were based on gender; $60.0 \%$ on race or ethnicity; $28.0 \%$ based on "other". Women respondents were 6.2 times more likely than men to report experiences of discrimination by patients and families. Black/AA respondents were 3.2 times more likely than White respondents to report experiencing discrimination by patients and families.

Moreover, our survey inquired about observed offensive remarks made towards patients or families. Sixty-three percent of participants indicated witnessing such remarks. Lastly, when asked if respondents thought there were disparities in the care given to patients of different ethnic groups at their hospital/clinical location, $58.3 \%$ of respondents agreed, $13.9 \%$ of them did not know, and $27.8 \%$ disagreed.

\section{Institutional Support Structures}

Participants were asked if they were aware of whether their institution offered a mechanism to address discrimination from patients and/or families: $32.7 \%$ indicated mechanisms were present, $47.6 \%$ did not know, and $19.6 \%$ indicated no mechanism. Similarly, participants indicated that $36.1 \%$ knew what to do if faced with discriminatory behaviors from patients and/or family, 30.6\% did not know, and 33.3\% did not know what to do.
When examining these responses by race, Black/AA respondents were least likely to think that their institution would respond appropriately to reports of discrimination. Black/AA respondents were also less likely than White respondents to agree with the statement that they would recommend their institution as supportive for people of color.

\section{Discussion}

This study adds to existing knowledge about URG trainees through our research questions: Do internal medicine trainees perceive their learning environment, programmatic and institutional structures responsive to issues of racism, bias, and discrimination? To what extent do residents from URGs perceive their learning environment to be welcoming and inclusive?

First, our findings suggest that trainees perceive a gap in formal mechanisms for reporting, an important first step in dealing with instances of discrimination and racism programmatically and institutionally. The absence of such mechanisms is especially problematic within the context of the current era and frankly, a global movement towards not just diverse and equitable learning environments, but towards inclusive, antiracist and anti-oppressive environments. Racism, bias, discrimination, and microaggressions are perpetrated by individuals but also perpetuated through complicit silence, flawed infrastructures, lack of leadership, institutional bias, and so forth. We urge programs to evaluate and enact changes that allow trainees to report these instances and feel safe in doing so while also making 
visible the process for reporting, and transparent about the consequences for perpetrators.

Our study indicated that $63 \%$ percent of participants indicated witnessing offensive remarks from the healthcare team towards patients from diverse backgrounds. Moreover, 58\% perceived disparities in the health care given to patients of different ethnic groups at their hospital/clinical location. A fair and transparent reporting system would allow for such reporting. This has a serious impact on the ability to address health equity in a system plagued with racial and ethnic disparities and health outcomes. Health equity is the goal of academic medicine. We strive for a state in which everyone has the opportunity to attain full health and individuals are not disadvantaged because of perceived memberships to minoritized groups in a society. Lastly, the fact that our URG trainees are seeing how the health system treats their own, how can we pretend that our learning environments are safe, welcoming, and a place where they should feel they belong.

Like other scholars, this study confirms prior arguments that residency programs must shift their structures and culture to better foster a supportive environment for women trainees. ${ }^{12}$ However, in the interest of intersectional identities, the most salient racial relationship suggested that Black/AA were 9.5 times more likely to report needing to minimize aspects of their race including language, clothes, style, and ways of interacting with others in order to be able to "fit in" in medicine. Our system is perpetuating assimilation to an image perceived as the exemplar of what a physician looks like. The problem with fitting in is its subjectivity determined by a majority group and its exclusionary and oppressive consequences. Fitting in may discourage reporting of instances, may make URGs feel less than their authentic selves.

Several important implications for research, practice, and policy emerge from this study. First, our survey tool demonstrates feasibility in gathering this critical information and future research will broaden the utilization of this survey tool. Second, robust clinician educator professional development appears to be needed. For example, learning and practicing how to respond to instances of racism, sexism, discrimination, and microaggressions should be incorporated into residency education and faculty development. ${ }^{30}$ Third, as institutional policies are developed, they should track, report, and respond to incidents in a transparent manner.

Lastly, we would be remiss to mention the current opportunity that institutions have to correct the past and move forward towards truly embracing equality, equity, diversity, inclusion, and anti-oppression in ways that are far from performative in nature. As leaders in academic medicine, we can lead our institutions to exemplars of clinical education while owning our social responsibility and living its mission. It is time for a deep reflection of the type of institution and the type of educators we want to be, on behalf of our trainees. Box 1 offers a series of recommendations for creating inclusive environments and addressing bias and discrimination against trainees.

\section{Limitations}

The main goal of this survey was to assess the learning environments of IM residents. Through this exercise, we encountered limitations in the feasibility of IM trainees as participants given the nature and intensity of their training and obvious time constraints. However, this is an opportunity for institutions and programs to learn more about their own practices. By requiring this type of learning environment assessment, institutions can intervene early, address intentionally these issues, while using a tracking tool that demonstrates a commitment to action.

\section{Conclusion}

Existing research indicates that URGs routinely deal with racism, bias, discrimination, and microaggressions. Having

Box I Recommendations for Creating an Inclusive Environment and Addressing Bias and Discrimination Against Trainees

Recommendations for Creating an Inclusive Environment and Addressing Bias and Discrimination Against Trainees

Conduct a learning environment review on an annual basis to establish a baseline and measure changes over time

Implement confidential and meaningful reporting structures and ensure that all trainees are aware of them

Maintain a structure for addressing bias and discrimination and following up with trainees with transparency throughout the organization

Ensure no tolerance policies for bias and discrimination events Faculty development to assist trainees and navigate the process with residents must be disseminated

Clear and visible commitment to promote health equity and mitigate disparities for patients at risk must be baked into the quality strategy for training programs and their sponsoring institutions 
access to timely information about the experiences of discrimination and bias that exist in the graduate medical education learning environment is imperative to disrupt systemic issues, along with health disparities and inequities. Residency and training periods are marked by significant stress and IM programs need to do better at acknowledging these challenges, address them not only at the programmatic level but embrace the agency they have to address institutional bias and systemic attitudes towards a diverse workforce.

\section{Disclosure}

The authors report no conflicts of interest in this work.

\section{References}

1. Komaromy M, Grumbach K, Drake M, et al. The role of black and Hispanic physicians in proving health care for underserved populations. N Engl J Med. 1996;334(20):1305-1310. doi:10.1056/ NEJM199605163342006

2. King TE, Dickinson TA, DuBose TD, et al. The case for diversity in academic internal medicine. Am J Med. 2004;116(4):284-289. doi:10.1016/j.amjmed.2003.12.015

3. Cohen JJ, Gabriel BA, Terrell C. the case for diversity in the health care workforce. Health Aff. 2002;21(5):90-102. doi:10.1377/ hlthaff.21.5.90

4. DeLisa JA, Lindenthal JJ. Commentary: reflections on diversity and inclusion in medical education. Acad Med. 2012;87(11):1461-1463. doi:10.1097/ACM.0b013e31826b048c

5. Marrast LM, Zallman L, Woolhandler S, et al. Minority physicians' role in the care of underserved patients: diversifying the physician workforce may be key in addressing health disparities. JAMA Int Med. 2014;174(2):289-291. doi:10.1001/jamainternm ed.2013.12756

6. Saha S. Taking diversity seriously: the merits of increasing minority representation in medicine. JAMA Int Med. 2014;174(2):291-292. doi:10.1001/jamainternmed.2013.12736

7. Nivet MA. Minorities in medicine: review of the literature. $J$ Vasc Surgery. 2010;51(4):53S-58S. doi:10.1016/j.jvs.2009.09.064

8. Nunez-Smith M, Curry LA, Bigby JA, et al. Impact of race on the professional lives of physicians of African descent. Ann Intern Med. 2007;146(1):45-51. doi:10.7326/0003-4819-146-1-200701020-00008

9. Cook DJ, Liutkus JF, Risdon CL, et al. Residents' experiences of abuse, discrimination and sexual harassment during residency training. Can Med Assoc J. 1996;154(11):1657.

10. Liebschultz JM, Darko GO, Finley EP. In the minority: black physicians in residency and their experiences. $J$ Natl Med Assoc. 2006;98 (9):1441-1448.

11. Lee KP, Kelz RR, Dube B, Morris JB. Attitudes and perceptions of the other underrepresented minority in surgery. J Surg Educ. 2014;71 (6):e47-e55. doi:10.1016/j.jsurg.2014.05.008

12. Woods SE, Harju A, Rao S, et al. Perceived bias and prejudices experienced by international medical graduates in the U.S. post-graduate medical education system. Med Educ Online. 2006;11 (1):20.
13. Fnais N, Soobiah C, Chen MH. Harrassment and discrimination in medical training. A systematic review and meta-analysis. Acad Med. 2014;89(5):817-824). doi:10.1097/ACM.0000000000000200

14. Tolbert CA, Rk K. Workplace discrimination: experiences of practicing physicians. J Natl Med Assoc. 2005;97(4):467-477.

15. Hassouneh D, Lutz KF, Beckett AK, et al. The experiences of underrepresented minority faculty in schools of medicine. Med Educ Online. 2014;19(1):24768 doi:10.3402/meo.v19.24768

16. Pololi L, Cooper LA, Carr P. Race, disadvantage and faculty experiences in academic medicine. $J$ Gen Intern Med. 2010;25 (12):1363-1369. doi:10.1007/s11606-010-1478-7

17. Sue DW, Capodilupo CM, Torino GC, et al. Racial microaggressions in everyday life: implications for clinical practice. Am Psychol. 2007;62(4):271-286. doi:10.1037/0003-066X.62.4.271

18. Sotto-Santiago $\mathrm{S}$ What gets lost in the numbers: a case study of the experiences and perspectives of black and latino faculty in academic medicine. [Dissertation]. Denver: University of Denver; 2017.

19. Bruce AN, Battista A, Plankey MW. Perceptions of gender-based discrimination during surgical training and practice. Med Educ Online. 2015;20(1):25923 doi:10.3402/meo.v20.25923

20. Osseo-Asare A, Balasuriya L, Huot SJ, et al. Minority resident physicians' views on the role of race/ethnicity in their training experiences in the workplace. JAMA Netw Open. 2018;1(5): e182723. doi:10.1001/jamanetworkopen.2018.2723

21. Dyrbye LN, Thomas MR, Eacker A, et al. Race, ethnicity, and medical student well-being in the United States. Arch Intern Med. 2007;167(19):2103-2109. doi:10.1001/archinte.167.19.2103

22. Boatright D, Branzetti J, Duong D, et al. Racial and ethnic diversity in academic emergency medicine: how far have we come? Next steps for the future. AEM Educ Train. 2018;2(Suppl Suppl 1):S31-S39. doi:10.1002/aet2.10204

23. NRMP. Residency Match found at ; 2021. Available from: https:// mk0nrmp3oyqui6wqfm.kinstacdn.com/wp-content/uploads/2021/03/ Advance-Data-Tables-2021_Final.pdf. Accessed November 4, 2021.

24. Dressel E. As doctor shortage continues, residency programs show some success at graduating more primary care physicians. Johns Hopkins Medicine Newsroom; April 9, 2019. Available from: https://www.hopkinsmedicine.org/news/newsroom/news-releases/asdoctor-shortage-continues-residency-programs-show-some-successat-graduating-more-primary-care-physicians. Accessed June 16, 2021.

25. Museus SD, Jayakumar UM. Creating Campus Cultures: Fostering Success Among Racially Diverse Student Populations. New York: Routledge; 2012.

26. Fowler FJ. Survey Research Methods. Sage publications; 2013.

27. Johanson GA, Brooks GP. Initial scale development: sample size for pilot studies. Educ Psychol Meas. 2010;70(3):394-400. doi:10.1177/ 0013164409355692

28. Gliner JA, Morgan GA, Leech NL. Research Methods in Applied Settings: An Integrated Approach to Design and Analysis. 2nd ed. Routledge/Taylor \& Francis Group; 2009.

29. Fosnacht K, Sarraf S, Howe E, Peck L. How important are high response rates for college surveys? Rev High Educ. 2017;40 (2):245-265. doi:10.1353/rhe.2017.0003

30. Sotto-Santiago S, Mac J, Duncan F, Smith J. "I didn't know what to say.": responding to racism, discrimination, and microaggressions with the OWTFD approach". Meded Portal. 2020. doi:10.15766/ mep_2374-8265.10971 


\section{Publish your work in this journal}

Advances in Medical Education and Practice is an international, peerreviewed, open access journal that aims to present and publish research on Medical Education covering medical, dental, nursing and allied health care professional education. The journal covers undergraduate education, postgraduate training and continuing medical education including emerging trends and innovative models linking education, research, and health care services. The manuscript management system is completely online and includes a very quick and fair peer-review system. Visit http://www.dovepress.com/testimonials.php to read real quotes from published authors. 